\title{
Tocilizumab-Induced Unexpected Increase of Several Inflammatory Cytokines in Critically III COVID-19 Patients: The Anti-Inflammatory Side of IL-6
}

\author{
Fanny Ponthieux,,i Nicolas Dauby, ${ }^{2,3}$ Evelyne Maillart, ${ }^{4}$ Jean-François Fils, ${ }^{5}$ Julie Smet, ${ }^{1}$ Marc Claus, ${ }^{6}$ \\ Tatiana Besse-Hammer, ${ }^{7}$ David De Bels, ${ }^{8}$ Francis Corazza, ${ }^{1,9}$ and Carole Nagant ${ }^{1}$
}

\begin{abstract}
Early evidence during the coronavirus disease 2019 (COVID-19) pandemic indicated high levels of interleukin (IL)-6 in patients with severe COVID-19. This led to the off-label use of tocilizumab (TCZ) during the first wave of the pandemic. While the monoclonal antibody blocks IL-6 pathway, its effect on other inflammatory cytokines remains poorly described. To better understand the effect of TCZ on the biological inflammatory profile, we monitored a large panel of inflammatory cytokines in critically ill COVID-19 patients receiving offlabel TCZ. Twenty-three patients with polymerase chain reaction-confirmed severe acute respiratory syndrome coronavirus 2 (SARS-CoV-2) infection were included in the study, among which 15 patients received TCZ and 8 patients did not. Serum samples were collected for 8 days, before and following TCZ administration or hospital admission for the control group. Serum profile of 12 cytokines (IL-1 $\beta,-2,-4,-6,-8,-10,-12,-13,-17$, -18 , tumor necrosis factor $\alpha$ (TNF- $\alpha$ ), interferon-gamma (IFN- $\gamma$ ), and sIL-6R were assessed in these two groups. Although the increased IL-6 concentrations after TCZ infusion were expected, we observed an unexpected increase in IL-1 $\beta,-2,-4,-10,-12 p 70,-18$, and sIL-6R levels in the treated patients with maximal values reaching 2 to 4 days after TCZ. In contrast, no change in cytokine levels was observed in the control group. Our results suggested that some inflammatory pathways escape IL-6R blockade and even appeared amplified. This finding highlights an old observation of the anti-inflammatory effects of IL- 6 as already suggested over 20 years ago. Clinical Trial Registration number: NCT04346017.
\end{abstract}

Keywords: inflammation, cytokines, IL-6, tocilizumab, COVID-19, SARS-CoV-2 infection

\section{Introduction}

$\mathbf{M}$ ORE THAN 30 YEARS have passed since discovery, cloning, and understanding of the biological role of interleukin-6 (IL-6) (3). The IL-6 signaling and its role in pathologies is currently well established and follow two different pathways involving the IL-6 receptor (IL-6R) and the glycoprotein130 (gp130) (3). The role of IL-6 dysregulation in various autoimmune and inflammatory diseases has been the rationale to target its pathway and led to the first antibody designed to block IL-6R, Tocilizumab (TCZ) $(6,12,23)$.

\footnotetext{
${ }^{1}$ Immunology Department, LHUB-ULB, Université libre de Bruxelles (ULB), Brussels, Belgium.

Departments of ${ }^{2}$ Infectious Diseases and ${ }^{6}$ Intensive Care, Centre Hospitalier Universitaire Saint-Pierre, Université libre de Bruxelles (ULB), Brussels, Belgium.

${ }^{3}$ Institute for Medical Immunology, Université libre de Bruxelles (ULB), Brussels, Belgium.

${ }^{4}$ Department of Infectious Disease, ${ }_{7}^{7}$ Clinical Research Department, and ${ }^{8}$ Department of Intensive Care, Brugmann University Hospital, Brussels, Belgium.

${ }_{9}^{5}$ Ars Statistica, Brussels, Belgium.

${ }^{9}$ Laboratory of Translational Research, Brugmann University Hospital, Université libre de Bruxelles (ULB), Brussels, Belgium.

i ORCID ID (https://orcid.org/0000-0002-9831-2218).

An earlier draft of this article was posted as a preprint at Research Square (DOI: 10.21203/rs.3.rs-234733/v1).
} 
TCZ is a humanized monoclonal antibody directed against both the soluble form and membrane-bound IL-6R and blocks the IL-6 signal pathway through competitive inhibition of the receptor $(12,20,23,31)$. It was first introduced in Japan in 2008 followed by the European Union in 2009, under the name of RoActemra ${ }^{\circledR}$, and the United States in 2010 as Actemra $^{\circledR}$, now licensed for use in more than 100 countries for treatment of rheumatoid arthritis, juvenile idiopathic arthritis, or Castleman's disease $(6,23,31)$.

From the beginning of the current pandemic, TCZ has been proposed in an off-label application to manage critical cases of coronavirus disease 2019 (COVID-19) and has quickly been included in the Chinese treatment guidelines $(22,32)$. Numerous clinical trials are ongoing to determine the most relevant target and therapeutic strategy in the management of COVID-19. TCZ is the purpose of ongoing nationwide (COV-AID), European (TOCIVID-19 in Italy, TOCOVID-19 in Spain), and international clinical trials (COVIDOSE in United States, TACOS in China), registered on clinicaltrials.gov. The optimal moment of administration for maximal therapeutic effect and the efficacy and safety of TCZ is still under investigation $(7,14,25)$. Furthermore, the genuine effect of this drug on the cytokine storm remains poorly described $(15,25)$.

In this context, we aimed to monitor IL-6 and several other inflammatory cytokines in critically ill COVID-19 patients before and during 8 days following anti-IL-6 therapy administration.

\section{Materials and Methods}

\section{Study design}

Overall, 23 patients admitted to the CHU Saint-Pierre and Brugmann (Brussels, Belgium) between 18 March and May 6, 2020 with a confirmed diagnosis of COVID-19 by a realtime polymerase chain reaction assay on a nasopharyngeal swab were enrolled in our study.

Patients were classified as severe or critical according to WHO guidelines 2020 as follows:

- Severe cases that met any of the following criteria: respiratory distress ( $\geq 30$ breaths/min); oxygen saturation $\leq 93 \%$ at rest; arterial partial pressure of oxygen $\left(\mathrm{PaO}_{2}\right)$ /fraction of inspired oxygen $\left(\mathrm{FiO}_{2}\right) \leq 300 \mathrm{mmHg}$ $(1 \mathrm{mmHg}=0.133 \mathrm{kPa})$.

- Critical cases that met any of the following criteria: respiratory failure and requiring mechanical ventilation; shock; other organ failure that requires intensive care unit (ICU) care.

Demographics data and clinical outcomes were collected from the medical file.

Fifteen patients received TCZ and were further divided in two groups, patients who survived (TCZ/S, $n=6)$ and nonsurvivors (TCZ/D, $n=9)$. We performed serum sample collection and cytokine measurements before TCZ infusion, day 0 , and during the 8 days following. In parallel, cytokine levels were measured at the admission, time identified as day 0 , and during the following 8 days in 8 COVID-19positive patients who did not receive TCZ (control group, NO TCZ). Each cytokine was measured three times (median, range 2-5) during the follow-up time post-TCZ or postadmission (Table 1).
In the time frame of this study, all affected patients were treated with the institutional standard pharmacological protocol, including $2,400 \mathrm{mg}$ of hydroxychloroquine in monotherapy over 5 days (4). When given, TCZ was administrated in a single intravenous dose of $8 \mathrm{mg} / \mathrm{kg}$, except for one patient who benefitted from a second one 3 days after. Patients did not receive corticosteroids or others immunomodulatory drugs.

The study was approved by the local Ethics Committee of the CHU Brugmann (CE 2020/63) and Saint-Pierre (Reference CE/20-03-05) Hospitals and registered on clinicaltrials.gov.

\section{Cytokine measurements}

The levels of 12 cytokines (IL- $1 \beta,-2,-4,-6,-8,-10,-12$, $-13,-17,-18$, tumor necrosis factor $\alpha[\mathrm{TNF}-\alpha]$, and interferon-gamma [IFN- $\gamma]$ ) and of the sIL-6R were assessed in the serum of patients using an electrochemiluminescence plate-based assay (MSD, Meso Scale Discovery, MD).

\section{Laboratory findings}

Circulating inflammatory biomarker levels (C-reactive protein $[\mathrm{CRP}]$, procalcitonin $[\mathrm{PCT}]$, ferritin, fibrinogen, d-dimers, and neutrophils) were measured before TCZ administration and for 8 days following the treatment or admission using a Cobas 8000 Platform (Roche Diagnostics), a Sysmex CS-5100 analyzer (Siemens Healthcare Diagnostics), and a Lumipulse G600II analyzer (Fujirebio).

\section{Statistical analysis}

Demographic data were analyzed using the nonparametric Kruskal-Wallis analysis of variance, followed by a Dunn's multiple comparison test. Categoric variables were compared with the Fisher's exact test.

Linear mixed models were used to analyze the longitudinal evolution of cytokine levels (29). For all models, the next effects were tested to model the evolution of biological variables through time: a group effect and a time effect. When the residuals of the model were not normally distributed, we used the bestNormalize $\mathrm{R}$ package to transform the outcome and reported the results of this last linear mixed model.

\section{Results}

Fifteen patients receiving TCZ (survivors, TCZ/S group, $n=6$ and nonsurvivors, TCZ/D group, $n=9$ ) and eight controls were followed for cytokine measurements.

Demographic data are reported in Table 1. There was no statistically significant difference according to the sex, the age, and the state of illness between subgroups. The median delay time between symptom onset and hospital admission was of 7 days in the three groups. The median (min-max) time before administration of TCZ was of 9 days (5-23) since symptoms emergence, 5 days (1-10) since hospital admission and 3 days (1-7) since ICU admission, without significant difference between the TCZ/S and TCZ/D group (Table 1).

Cytokine measurements assessed in each patient are listed in Table 2. 
Table 1. Demographic and Clinical Characteristics

\begin{tabular}{|c|c|c|c|c|c|c|}
\hline Variable & $\begin{array}{c}N O T C Z \\
(N)(\mathrm{n}=8)\end{array}$ & $\begin{array}{l}\text { TCZ/nonsurvivors } \\
\text { (D) }(\mathrm{n}=9)\end{array}$ & $\begin{array}{l}\text { TCZ/survivors } \\
(S)(\mathrm{n}=6)\end{array}$ & $\frac{\mathrm{p} \text {-value }}{N-D}$ & $\frac{\mathrm{p} \text {-value }}{N-S}$ & $\frac{\mathrm{p} \text {-value }}{D-S}$ \\
\hline Age, years & 70 [31-91] & $65[44-84]$ & $48.5[41-74]$ & ns & ns & ns \\
\hline \multicolumn{7}{|l|}{ Gender } \\
\hline Male & $6(75 \%)$ & $7(77.8 \%)$ & $4(66.7 \%)$ & \multirow[t]{2}{*}{ ns } & \multirow[t]{2}{*}{ ns } & \multirow[t]{2}{*}{ ns } \\
\hline Female & $2(25 \%)$ & $2(22.2 \%)$ & $2(33.3 \%)$ & & & \\
\hline \multicolumn{7}{|l|}{ State of illness } \\
\hline Severe & $3(37.5 \%)$ & - & - & \multirow[t]{2}{*}{ ns } & \multirow[t]{2}{*}{ ns } & \multirow[t]{2}{*}{ - } \\
\hline Critical & $5(62.5 \%)$ & $9(100 \%)$ & $6(100 \%)$ & & & \\
\hline \multicolumn{7}{|l|}{ Comorbidities } \\
\hline Diabetes & $2(25 \%)$ & $5(55.6 \%)$ & $1(16.7 \%)$ & ns & ns & ns \\
\hline Hypertension & $6(75 \%)$ & $5(55.6 \%)$ & $1(16.7 \%)$ & ns & ns & ns \\
\hline Dyslipidemia & $3(37.5 \%)$ & $1(11.1 \%)$ & $1(16.7 \%)$ & ns & ns & ns \\
\hline Cardiovascular disease & $2(25 \%)$ & $3(33.3 \%)$ & $0(0 \%)$ & ns & ns & ns \\
\hline Chronic kidney disease & $1(12.5 \%)$ & $2(22.2 \%)$ & $0(0 \%)$ & ns & ns & ns \\
\hline Chronic pulmonary disease & $2(25 \%)$ & $0(0 \%)$ & $1(16.7 \%)$ & ns & ns & ns \\
\hline $\begin{array}{l}\text { Number of cytokine } \\
\text { measurements }\end{array}$ & $2.5[2-3]$ & $4[2-5]$ & $3.5[2-5]$ & ns & ns & ns \\
\hline \multicolumn{7}{|l|}{ Hospitalization time } \\
\hline Total, days & $43.5[5-137]$ & $25[5-57]$ & $53.5[24.0-134]$ & ns & ns & ns \\
\hline ICU, days & $7.5[0-49]$ & $27[11-56]$ & $44[12-65]$ & ns & ns & ns \\
\hline \multicolumn{7}{|l|}{ Interval time between, days } \\
\hline Symptoms and admission & $7[1-21]$ & $7[4-14]$ & $7[1-13]$ & ns & ns & ns \\
\hline Symptoms and ICU admission & $10.5[4-15]$ & $9[5-15]$ & $11.5[1-15]$ & ns & ns & ns \\
\hline Symptoms and TCZ infusion & - & $11[8-23]$ & $14[11-18]$ & - & - & 0.1046 \\
\hline Admission and TCZ infusion & - & $4[1-5]$ & $6[4-10]$ & - & - & 0.0713 \\
\hline ICU admission and TCZ infusion & - & $2[0-8]$ & $3.5[1-10]$ & - & - & 0.2849 \\
\hline
\end{tabular}

Data are expressed as median [min-max] or $n(\%)$.

ICU, intensive care unit; ns, non significant; TCZ, tocilizumab.

The baseline and maximum levels obtained for each cytokine were compared among patient groups and reported in Table 3. No difference was observed in cytokine baseline levels between groups, except for IL-2 and IL-6, which were found to be higher in treated patients before treatment administration. IL- 6 concentrations rapidly raised and reached significantly higher maximum levels in TCZ-treated patients compared with the control group.

Moreover, significantly higher levels of IL- $1 \beta,-2,-4,-6 \mathrm{R}$, $10,-12 p 70$, and -18 were also observed in patients receiving TCZ. These levels were elevated in both the TCZ/S group and the TCZ/D group, except for IL-4 and -10 , which reached significantly higher values only in the TCZ/D group when compared with the control group and for sIL-6R. However, no statistically significant difference was observed in maximal values between the TCZ/S group and the TCZ/D group for the different cytokines on our studied population (Table 3).

Cytokine profiles, assessed before and 8 days after TCZ administration, are shown in Figure 1. Longitudinal changes in cytokine levels were analyzed using a linear mixed model and showed a significant transient effect (time ${ }^{2}$ effect) over time with a peak value that reached around day 2 to 4 for IL-2 (only in TCZ/D), -4, -6, -6R (only in TCZ/S), -12p70, -13 , and TNF $\alpha$ (only in TCZ/D). Furthermore, the time associated with the maximal value for all cytokines was not significantly different among the three subgroups (TCZtreated and nontreated patients) (Table 3).

The laboratory parameter baseline results revealed high levels of inflammatory markers, including CRP, ferritin, fibrinogen, and neutrophils, for all patients enrolled. Most of them remained stable in the nontreated group during the 8 days of follow-up. In the treated patients, CRP and fibrinogen levels improved and decreased toward the normal range, whereas neutrophils and ferritin remained high above normal values, and d-dimers even increased in the TCZ/D group (Fig. 2).

\section{Discussion}

The cytokine profile of some critically ill COVID-19 patients shares similarities to those observed in cytokine release syndromes, characterized by an increased production of cytokines followed by a dysregulation of tissue repair mechanisms, damages and, finally, multiple organ failure leading to death $(5,19,28,32)$.

Recently, levels of IL- 6 have been included to the criteria for COVID-19-associated hyperinflammatory syndrome (30). Cytokines IL-6 and TNF- $\alpha$ have revealed to predict severity and mortality in COVID-19 (9). Although the mechanisms leading to inflammation in COVID-19 are still poorly understood, immunomodulatory agents have rapidly been proposed and included into various clinical trials, most of them focused on IL- $1 \beta,-6,-8$, and TNF- $\alpha(6,9,14)$. Azmy et al. suggested that TCZ was unable to calm the inflammatory storm focusing on increased IL-10 and IL-2 receptor levels in patients receiving TCZ (2). However, the profile of a large panel of cytokines immediately after TCZ administration is yet unknown. 


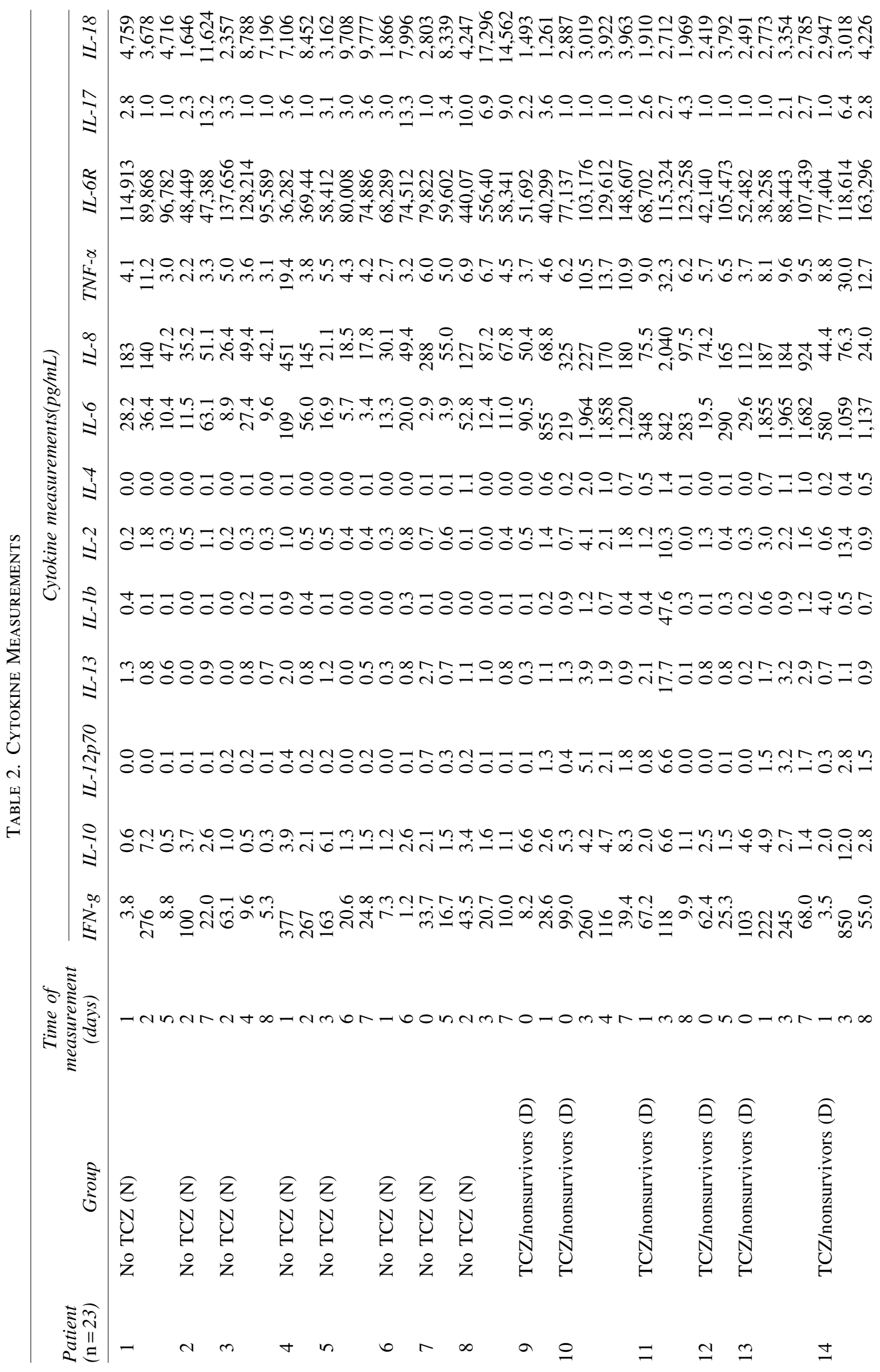




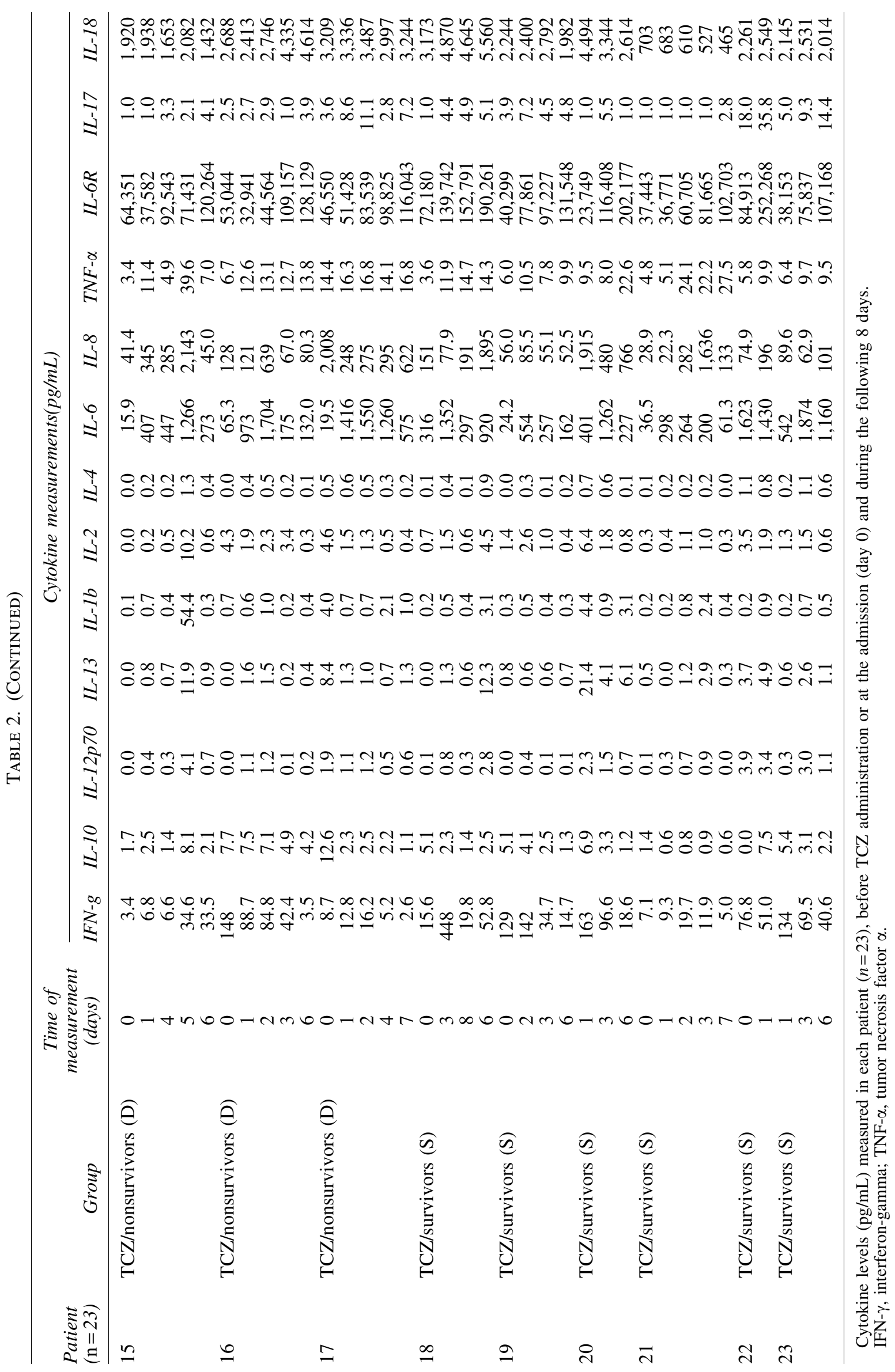




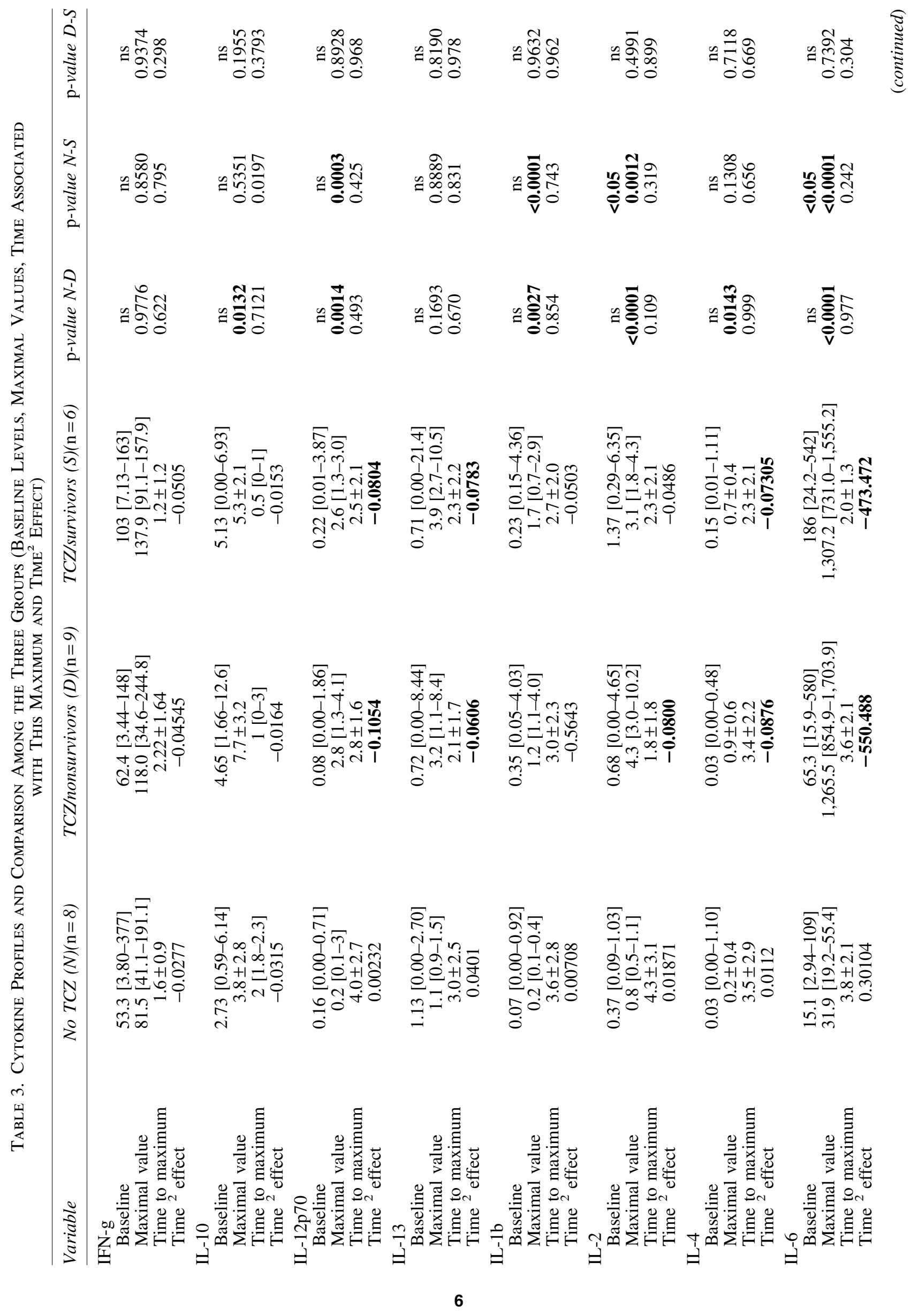




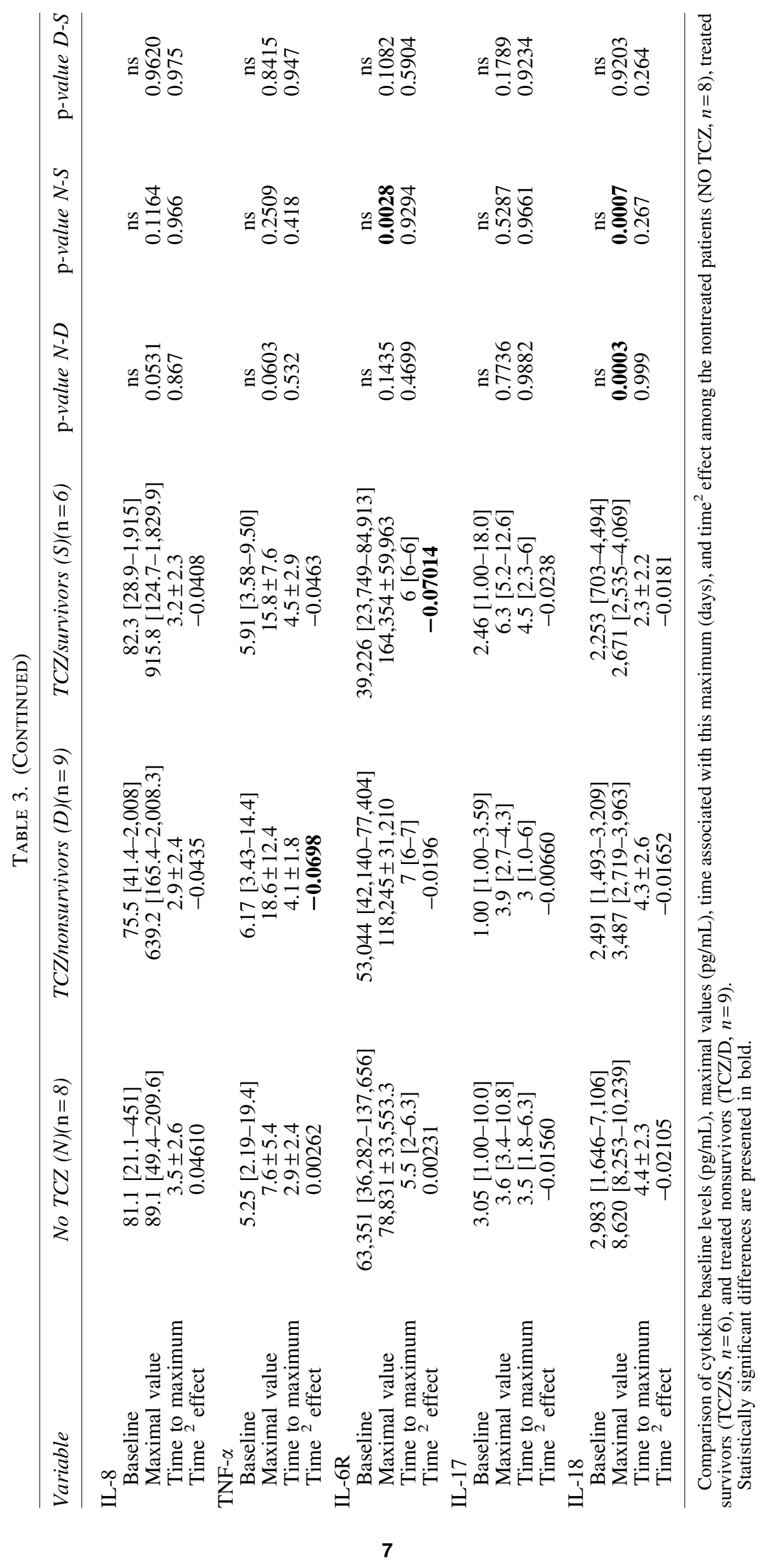



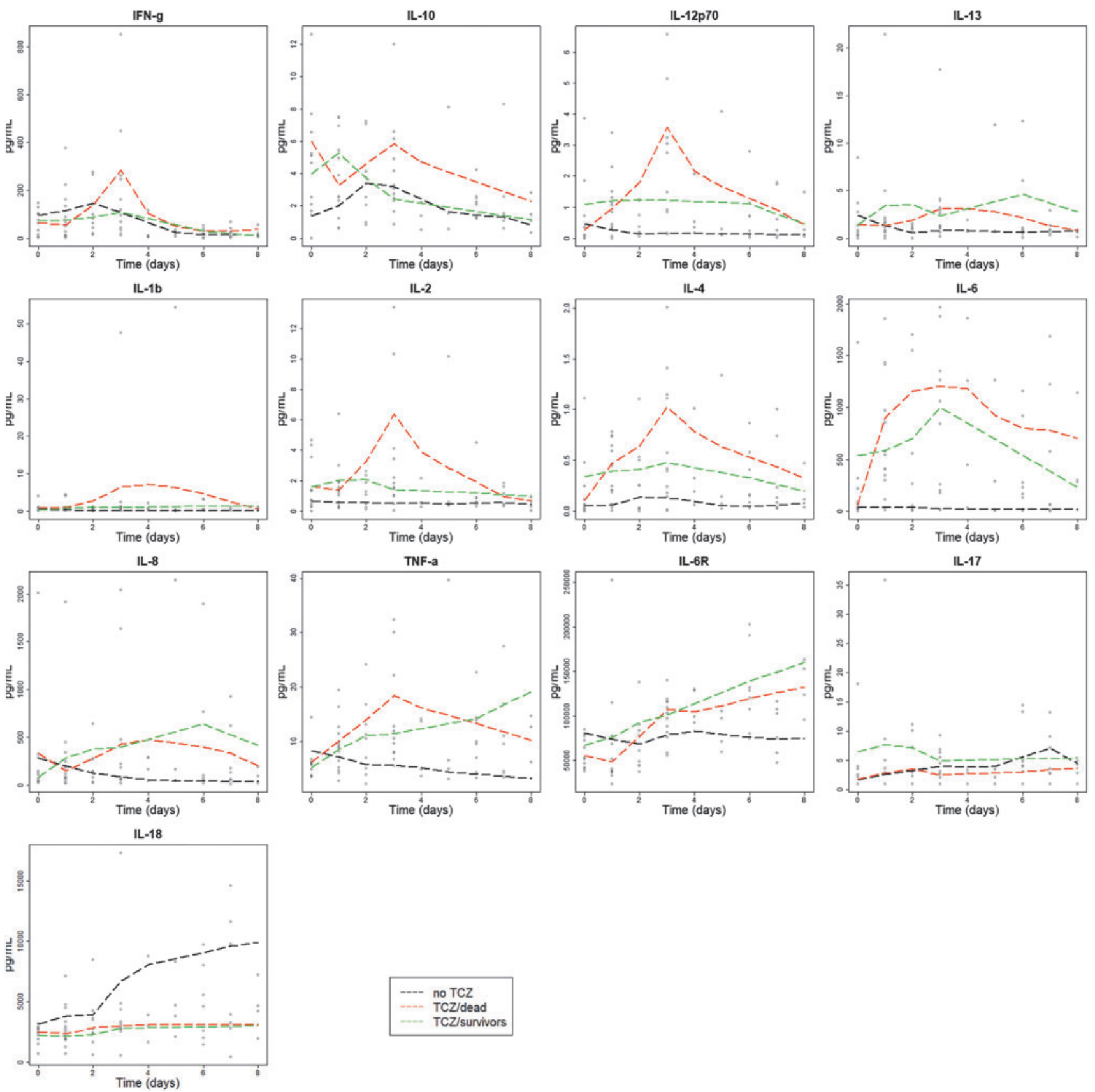

FIG. 1. Kinetics of cytokines in the three groups. Kinetics of IL-1 $\beta,-2,-4,-6,-8,-10,-12,-13,-17,-18$, TNF- $\alpha$, and IFN- $\gamma$ in nontreated (NO TCZ, $n=8$ ) versus treated survivors (TCZ/S, $n=6)$ or treated nonsurvivors $(\mathrm{TCZ} / \mathrm{D}, n=9)$. Results were measured between day 0 and 8 after TCZ infusion, or from hospital admission for the NO TCZ group. IL, interleukin; TCZ, tocilizumab; TNF- $\alpha$, tumor necrosis factor $\alpha$. Color images are available online.

In this study, we explored the longitudinal cytokine profile in critically ill severe acute respiratory syndrome coronavirus 2 (SARS-CoV2)-infected patients receiving anti-IL6R therapy. We focused on the trend of 12 inflammatory cytokines (IL-1 $\beta,-2,-4,-6,-8,-10,-12,-13,-17$, -18 , TNF- $\alpha$, and IFN- $\gamma$ ) and of the sIL-6R in the treated group compared with patients without anticytokine therapy.

Regarding the longitudinal evolution, the levels of most of cytokines remained stable over time following the disease course in the nontreated group, supporting previously reported results (9). In contrast, IL-18 levels progressively increased during the follow-up in untreated patients. It is noteworthy that IL-18 has also been recommended as a biomarker for secondary hemophagocytic lymphohistiocytosis $(8,13)$. However, whether the increase in IL-18 levels observed in a few COVID-19 patients is a witness of a SARS-CoV2-induced HLH remains questionable.

The significant difference observed for the baseline levels of IL-6 and found only in TCZ-treated survivors may indicate that a high IL- 6 concentration before treatment is required to benefit from TCZ as observed by Galván-Román et al. (11).

In the treated patients, we confirmed the expected rapid increase of IL- 6 levels after TCZ injection followed by a 

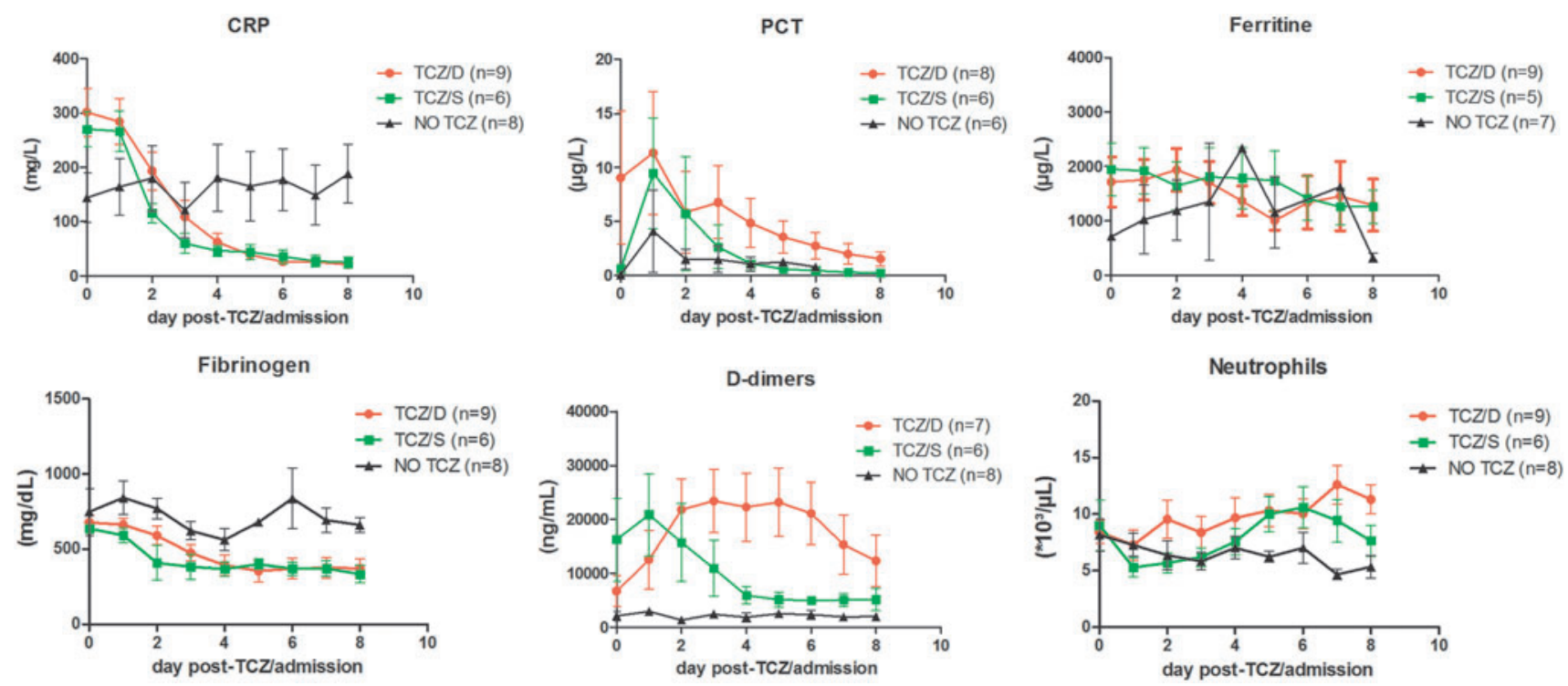

FIG. 2. Tocilizumab effect on circulating inflammatory biomarkers. Kinetics of CRP, PCT, ferritin, fibrinogen, d-dimer and neutrophil* levels in nontreated (NO TCZ, $n=8$ ) versus treated survivors (TCZ/S, $n=6$ ) and treated nonsurvivors $(\mathrm{TCZ} / \mathrm{D}, n=9)$. Results were measured between day 0 and 8 after TCZ infusion, or from hospital admission for the NO TCZ group. Results are presented as mean \pm SEM. *Not all patient received all relevant laboratory tests. CRP, C-reactive protein; PCT, procalcitonin; SEM, standard error of the mean. Color images are available online.

gradual decrease (9). This can be explained by the blockage of the IL-6R, resulting in the accumulation of IL-6, which can no longer bind to its receptor and thus cannot be internalized through IL-6R-mediated consumption $(14,31)$. This elevation also reflects the endogenous IL-6 production related to the disease activity (18).

In parallel, therapeutic inhibition of the IL-6R blocks the IL-6 signaling pathway but not the generation of sIL-6R, which is unaltered in TCZ-treated patients (20). The binding of TCZ to the IL-6R and accumulation of the resulting TCZ/ sIL-6R complexes might explain increased levels of IL-6R observed after TCZ injection as compared with the nontreated group since TCZ bound to sIL-6R has a higher molecular weight than sIL-6R alone and is therefore cleared more slowly from the circulation (Table 3).

More surprisingly, we also observed a transient but significant increase in proinflammatory cytokines: IL-2, -4, -612, -13 (only in TCZ/D), TNF- $\alpha$ (only in TCZ/D), and sIL-6R (only in TCZ/S), for patients receiving a single dose of IL-6R blockade therapy. This observation highlights that neutralization of the IL-6R pathway is not sufficient to completely prevent activation of all the inflammatory pathways. Our findings are in accordance with the Del Valle et al. (9) previous observations on TCZ effect on IL-6 and IL-8 levels, whereas we diverge about the profile of TNF- $\alpha$ presenting a transient increase following the treatment injection, as seen only in the TCZ/D group in our study (Table 3).

IL-6 signal transduction acts through both a soluble and a membrane-bound receptor involved in two different pathways with divergent functions: the classical one promoting antiinflammatory responses and the trans-signaling pathway responsible for the proinflammatory effects $(3,12,20,31,32)$. TCZ acts by blocking both and thus inhibits proinflammatory but also potentially protective anti-inflammatory IL-6-mediated responses (12).
In contrast to cytokines, circulating inflammatory biomarkers such as CRP, fibrinogen, and ferritin baseline levels were elevated but decreased shortly after TCZ administration, without any difference between survivor and nonsurvivor patients (Fig. 2). These laboratory findings corroborate with previous results of Toniati et al. (28), with a decrease of these biomarkers toward a normal range associated with a clinical improvement 10 days after TCZ. While the ferritin profile was stable over our follow-up time in all three groups, it remained above normal values. However, we noticed an increase in neutrophil and d-dimer levels in our treated population and especially in nonsurvivors, comforting that IL-6 pathway blockers act only partially on the inflammatory cascade.

Even if no statistically significant difference was observed in maximal values between the TCZ/S and the TCZ/D group for the different cytokines in our population, trends in increased levels of IL-12p70, IL-2, IL-4, IL-6, and TNF- $\alpha$ were noted in the TCZ patients who died. This group of patients also presented the highest d-dimers, neutrophils, and PCT at day 8 compared with other groups. Such a constellation allows supposition that disseminated intravascular coagulation with multisystem failure could have contributed to death in these patients and that TCZ failed to prevent this fatal issue.

In summary, on the one hand, some inflammatory biomarkers rapidly decreased in the 8 days following TCZ infusion and, on the other hand, levels of several inflammatory cytokines significantly increased after a single dose of TCZ, suggesting a partial effect of the drug on hyperinflammation. These findings enhance the pivotal role of the IL-6 pathway: it may have a downregulatory effect on several cytokines that disappears by blocking the binding of IL-6 to its receptor and thus resulting in an increased production of these cytokines. 
This incomplete inhibition of the inflammatory response, shortly after one dose of TCZ, might explain why most clinical trials failed to meet their primary endpoints of improved clinical status in COVID-19 patients $(14,25)$. Our hypothesis is in accordance with the findings of Angriman et al., suggesting that this rebound expression of non-IL-6 cytokines could be detrimental, and might reflect an activation of collateral inflammatory pathways (1).

The increased levels of several inflammatory cytokines observed after a single TCZ administration in SARS-CoV-2 severely ill patients might also explain why immunomodulatory therapies with a wider range of targets proved to be more efficient, as suggested by the benefit of dexamethasone use in the preliminary results of the RECOVERY study (26). Promising recent data suggest that, combined to dexamethasone, TCZ could decrease the use of mechanical ventilation and the mortality, compared with standard of care $(10,17,24,26,31,21)$.

Finally, in January 2021, the REMAP-CAP trial released preliminary evidence that tocilizumab could reduce fatalities among severely ill Covid-19 patients having or have completed a course of corticosteroids, and the drug was added to the United Kingdom recommended list for COVID-19 treatment (27). The nationwide Belgian COVAID trial that compares combination of anti-IL-6 and IL-1 blockade in patients with COVID-19-associated hyperinflammatory syndrome will provide an answer about the clinical benefit of targeting multiple inflammatory pathways (16).

Our work features several limitations and particularly the limited number of patients included in the study, which may explain why we could not observe a difference between survivor and nonsurvivor TCZ-treated patients.

To our knowledge, we are the first to fully investigate the kinetics of this wide panel of cytokines before and after antiIL6R blocker while the recently published data focused on IL-6, IL-10, and IL-2R levels after TCZ administration (2).

Besides the predictable peak of IL- 6 concentration, we highlight an immune dysregulation worsening in patients receiving TCZ, as evidenced by the increase of several other cytokines, among them some proinflammatory and potentially harmful ones. This observation reinforces the need to use another immunomodulatory strategy in critically ill COVID-19 patients, particularly for patients with low IL-6 baseline levels.

Our findings suggest that the IL-6 inhibition alone may not be sufficient to completely block the hyperinflammatory state described in critically ill COVID-19 patients and may constitute an argument pleading for a treatment that target more broadly the various pathways of inflammation. These biological results could explain why most of clinical trials using TCZ alone failed to meet their primary endpoints of improved clinical status, whereas studies in which a positive effect of TCZ has been observed included corticosteroids $(4,10,22,27)$.

\section{Authors' Contributions}

Concept and design: C.N., F.C., F.P., and J.S. Analysis and interpretation of cytokine data: C.N., F.C., and J.-F.F. Patient enrollment and collection of clinical data: N.D., E.M., D.D.B., and T.B.-H. Writing original draft: F.P. and C.N. All authors critically reviewed the article.

\section{Acknowledgments}

The authors thank Asma Benslimane and Thao Tran Thi Thanh for their technical help. N.D. is a postdoctorate clinical master specialist of the F.R.S-FNRS.

\section{Author Disclosure Statement}

No competing financial interests exist.

\section{Funding Information}

No funding was received.

\section{References}

1. Angriman F, Ferreyro BL, Burry L, et al. Interleukin-6 receptor blockade in patients with COVID-19: placing clinical trials into context. Lancet Respir Med 2021;9:655664.

2. Azmy V, Kaman K, Tang D, et al. Cytokine profiles before and after immune modulation in hospitalized patients with COVID-19. J Clin Immunol 2021;41:738-747.

3. Calabrese LH, and Rose-John S. IL-6 biology: implications for clinical targeting in rheumatic disease. Nat Rev Rheumatol 2014;10:720-727.

4. Catteau L, Dauby N, Montourcy M, et al. Low-dose hydroxychloroquine therapy and mortality in hospitalised patients with COVID-19: a nationwide observational study of 8075 participants. Int J Antimicrob Agents 2020;56: 106144.

5. Chen LYC, Hoiland RL, Stukas S, et al. Confronting the controversy: interleukin-6 and the COVID-19 cytokine storm syndrome. Eur Respir J 2020;56:2003006.

6. Choy EH, De Benedetti F, Takeuchi T, et al. Translating IL-6 biology into effective treatments. Nat Rev Rheumatol 2020;16:335-345.

7. De Stefano L, Bobbio-Pallavicini F, Manzo A, et al. A "Window of Therapeutic Opportunity" for anti-cytokine therapy in patients with coronavirus disease 2019. Front Immunol 2020;11:572635.

8. Debaugnies F, Mahadeb B, Nagant C, et al. Biomarkers for early diagnosis of hemophagocytic lymphohistiocytosis in critically ill patients. J Clin Immunol 2021;41:658-665.

9. Del Valle DM, Kim-Schulze S, Huang HH, et al. An inflammatory cytokine signature predicts COVID-19 severity and survival. Nat Med 2020;26:1636-1643.

10. Furlow B. COVACTA trial raises questions about tocilizumab's benefit in COVID-19. Lancet Rheumatol 2020;2:e592.

11. Galván-Román JM, Rodríguez-García SC, Roy-Vallejo E, et al. IL-6 serum levels predict severity and response to tocilizumab in COVID-19: an observational study. J Allergy Clin Immunol 2021;147:72-80.

12. Garbers C, Heink S, Korn T, et al. Interleukin-6: designing specific therapeutics for a complex cytokine. Nat Rev Drug Discov 2018;17:395-412.

13. Kessel C, Vollenberg R, Masjosthusmann K, et al. Discrimination of COVID-19 from inflammation-induced cytokine storm syndromes using disease-related blood biomarkers. Arthritis Rheumatol 2021;73:1791-1799.

14. Khiali S, Khani E, and Entezari-Maleki T. A comprehensive review of tocilizumab in COVID-19 acute respiratory distress syndrome. J Clin Pharmacol 2020;60:1131-1146.

15. Leisman DE, Ronner L, Pinotti R, et al. Cytokine elevation in severe and critical COVID-19: a rapid systematic review, 
meta-analysis, and comparison with other inflammatory syndromes. Lancet Respir Med 2020;8:1233-1244.

16. Maes B, Bosteels C, De Leeuw E, et al. Treatment of severely ill COVID-19 patients with anti-interleukin drugs (COV-AID): a structured summary of a study protocol for a randomised controlled trial. Trials 2020;21:20-21.

17. Narain S, Stefanov DG, Chau AS, et al. Comparative survival analysis of immunomodulatory therapy for coronavirus disease 2019 cytokine storm. Chest 2021;159:933-948.

18. Nishimoto $\mathrm{N}$, Terao $\mathrm{K}$, Mima $\mathrm{T}$, et al. Mechanisms and pathologic significances in increase in serum interleukin-6 (IL-6) and soluble IL-6 receptor after administration of an anti-IL-6 receptor antibody, tocilizumab, in patients with rheumatoid arthritis and Castleman disease. Blood 2008; 112:3959-3964.

19. Pelaia C, Calabrese C, Garofalo E, et al. Therapeutic role of tocilizumab in sars-cov-2-induced cytokine storm: rationale and current evidence. Int J Mol Sci 2021;22:1-16.

20. Preniss1 N, Lokau J, Rose-John S, et al. Therapeutic blockade of the interleukin-6 receptor (IL-6R) allows sIL-6R generation by proteolytic cleavage. Cytokine 2019;114:1-5.

21. Salama C, Han J, Yau L, et al. Tocilizumab in nonventilated patients hospitalized with Covid-19 pneumonia. medRxiv 2020;10:20210203.

22. Sanders JM, Monogue ML, Jodlowski TZ, et al. Pharmacologic treatments for coronavirus disease 2019 (COVID-19): a review. JAMA 2020;323:1824-1836.

23. Sheppard M, Laskou F, Stapleton PP, et al. Tocilizumab (actemra). Hum Vaccines Immunother 2017;13:1972-1988.

24. Stone JH, Frigault MJ, Serling-Boyd NJ, et al. Efficacy of tocilizumab in patients hospitalized with Covid-19. N Engl J Med 2020;383:2333-2344.

25. Tang Y, Liu J, Zhang D, et al. Cytokine storm in COVID-19: the current evidence and treatment strategies. Front Immunol 2020;11:1-13.
26. The Recovery Collaborative Group. Dexamethasone in hospitalized patients with Covid-19. N Engl J Med 2021; 384:693-704.

27. The REMAP-CAP Investigators. Interleukin-6 receptor antagonists in critically ill patients with Covid-19. N Engl J Med 2021;384:1491-1502.

28. Toniati P, Piva S, Cattalini M, et al. Tocilizumab for the treatment of severe COVID-19 pneumonia with hyperinflammatory syndrome and acute respiratory failure: a single center study of 100 patients in Brescia, Italy. Autoimmun Rev 2020;19:102568.

29. Verbeke G, and Molenberghs G. Linear Mixed Models for Longitudinal Data, Springer Series in Statistics. New-York: Springer-Verlag, 2000.

30. Webb BJ, Peltan ID, Jensen P, et al. Clinical criteria for COVID-19-associated hyperinflammatory syndrome: a cohort study. Lancet Rheumatol 2020;2:e754-e763.

31. Wolf J, Rose-John S, and Garbers C. Interleukin-6 and its receptors: a highly regulated and dynamic system. Cytokine 2014;70:11-20.

32. Yarmohammadi A, Yarmohammadi M, Fakhri S, et al. Targeting pivotal inflammatory pathways in COVID-19: a mechanistic review. Eur J Pharmacol 2021;890:173620.

Address correspondence to: Ms. Fanny Ponthieux Immunology Department LHUB-ULB, Université libre de Bruxelles (ULB) Place van Gehuchten 4 Brussels 1020 Belgium

E-mail: fanny.ponthieux@lhub-ulb.be 